\title{
Insights from the Structure of Mycobacterium tuberculosis Topoisomerase I with a novel protein fold
}

\author{
Kemin Tan, ${ }^{1^{*}}$ Nan Cao, ${ }^{2}$ Bokun Cheng, ${ }^{3}$ Andrzej Joachimiak, ${ }^{1}$ Yuk-Ching Tse-Dinh ${ }^{2,4^{*}}$ \\ ${ }^{1}$ Structural Biology Center, Biosciences Division, Argonne National Laboratory, \\ Argonne, IL 60439, USA \\ ${ }^{2}$ Department of Chemistry and Biochemistry, Florida International University, Miami, FL \\ 33199, USA. \\ ${ }^{3}$ Department of Biochemistry and Molecular Biology, New York Medical College, \\ Valhalla, NY 10595, USA. \\ ${ }^{4}$ Biomolecular Sciences Institute, Florida International University, Miami, FL 33199, \\ USA. \\ ${ }^{*}$ Corresponding authors \\ E-mail: ktan@anl.gov (KT), ytsedinh@fiu.edu (YT) \\ Tel: 1-630-252-3948 (KT), 1-305-348-4956 (YT)
}




\title{
Key words
}

TB, TopA, protein fold, protein domains, crystallization

\author{
Abbreviations \\ MtTOP1, M. tuberculosis topoisomerase I \\ TB, tuberculosis
}




\section{Introduction}

Topoisomerases are needed in all organisms to maintain the optimal level of DNA supercoiling for physiological functions, and to resolve topological barriers encountered during processes of replication, transcription, recombination, and repair. ${ }^{1,2}$ In most organisms, multiple topoisomerases are present, and each cellular topoisomerase has specific roles associated with these physiological processes. ${ }^{3}$ The genome of the pathogen Mycobacterium tuberculosis encodes only one type I topoisomerase that catalyzes topological transitions by cutting and rejoining a singlestrand of DNA and one type II topoisomerase that carries out catalysis by cutting and rejoining a double-strand of DNA. The type II topoisomerase (DNA gyrase) of $M$. tuberculosis is the target of moxifloxacin, a fluoroquinolone antibacterial agent being utilized in a new tuberculosis (TB) drug combination regimen and found to be beneficial for reducing TB treatment duration. ${ }^{4}$ The only type I topoisomerase in M. tuberculosis (MtTOP1, Rv3646c) is a type IA topoisomerase that has been found to be essential for growth, and its depletion was shown to decrease survival following treatment with novobiocin and isoniazid. ${ }^{5}$ Each year, around 9 million people worldwide develop TB caused by Mycobacterium tuberculosis, accounting for 1.3 million deaths. MtTOP1 has been actively investigated as a target for novel TB drugs ${ }^{6-8}$ that are very much needed for the treatment of drug-resistant TB. The structure of MtTOP1 will be essential for structure-based rational drug discovery efforts.

Our early trials to obtain diffraction quality crystals of the full length MtTOP1 protein were unsuccessful. Constructs of the MtTOP1 N-terminal region corresponding to the N-terminal domains D1-D4 found in E. coli topoisomerase I (EcTOP1) crystal 
structures did not express well as soluble proteins. In a recently determined structure of full length EcTOP1 in complex with ssDNA ${ }^{9}$ (PDB ID: $\underline{4 R U L}$ ), the C-terminal region of EcTOP1 comprises three 4-Cys zinc ribbon domains and two zinc ribbon-like domains, and a modular ssDNA-binding mode was observed. This suggests that the C-terminal region of MtTOP1 may also contain multiple domains with repeated and conserved sequence motifs even though the sequences of EcTOP1 and MtTOP1 C-terminal regions are very different with essentially no homologies. We analyzed the structural aspects of the C-terminal region of MtTOP1. The recognition of individual domains in this region helped us redesign constructs for functional studies and enabled us to obtain diffraction quality crystals for structural characterization of MtTOP1. We report here the crystal structure of MtTOP1-704t with the N-terminal domains D1-D4 and C-terminal domain D5 (a.a. A2-T704) and its functional characterizations.

\section{Results}

\section{Analysis of C-terminal domains of MtTOP1}

MtTOP1 does not have easily recognizable sequence motifs (such as the repeated 4-Cys Zn-binding motifs present in EcTOP1) that can help divide the MtTOP1 C-terminal region into domains. Nevertheless, MtTOP1 does bear four GxxGPY sequence motifs ${ }^{10}$ in its C-terminal region. Based on the locations of the four GxxGPY repeats, we divided the region into four repeat domains (Fig. 1). A secondary structure prediction indicated that each domain comprises three or four $\beta$-strands and one $C$ terminal $\alpha$-helix. ${ }^{11}$ The predicted folding of the MtTOP1 C-terminal domains is different from the zinc ribbon domains or zinc ribbon-like domains found in EcTOP1 and 
Thermotoga maritima topoisomerase I. ${ }^{12}$ After the fourth or last C-terminal domain there is an additional long highly positively charged tail ( 26 amino acids) in MtTOP1. Besides the basic C-terminal tail, there are two more stretches of basic amino acids. ${ }^{13}$ Based on the prediction, one is a part of the insertion on the loop between the $\beta 3$ and $\beta 4$ strands of D6, and the other is a part of the linker between the D7 and D8 domains

(Fig. 1). Deletions of the stretches of basic amino acids in the C-terminal region of MtTOP1 have been shown to affect DNA binding and DNA strand passage. ${ }^{13}$ The deletions would unlikely make an impact on the folding of the individual C-terminal domains but may affect the relative orientations of the $\mathrm{C}$-terminal domains and the interdomain flexibility.

MtTOP1-704t is one of the constructs that have been made based on the Cterminal domain prediction above. It spans from amino acid A2 to T704 and includes the N-terminal domains and the first predicted C-terminal domain D5 (Fig.1 and Graphical Abstract). The functional and structural characterization of MtTOP1-704t is the focus of this study.

\section{Biochemical activities of MtTOP1-704t}

MtTOP1-704t can be expressed readily as a soluble recombinant protein in $E$. coli host strain T7 Express Crystal. The protein was purified to >99\% homogeneity and assayed for relaxation of negatively supercoiled plasmid DNA. The results in Fig. 2 showed that while relaxation activity can be detected with $6.2 \mathrm{ng}$ ( $60 \mathrm{fmole})$ of the full length MtTOP1, relaxation activity was not observed with $100 \mathrm{ng}$ (1.3 pmole) of MtTOP1-704t. 
Although relaxation activity could not be detected for MtTOP1-704t, the protein was found to have DNA cleavage activity similar to that of full length MtTOP1 (Fig. 3A). The same preferred cleavage site with a cytosine base that is four nucleotides upstream of the cleavage site was recognized for formation of the cleavage product. ${ }^{14}$ Intramolecular religation was promoted by adding $\mathrm{Mg}(\mathrm{II})$ to shift the cleavage-religation equilibrium toward religation along with $1 \mathrm{M} \mathrm{NaCl}$ to dissociate the non-covalent enzyme-DNA complex. The results in Fig. 3B showed that MtTOP1-704t is efficient in DNA religation, so the C-terminal sequences not found in MtTOP1-704t do not affect the cleavage and rejoining of ssDNA by MtTOP1.

There are two types of IA topoisomerases in most species of bacteria that have been studied. E. coli topoisomerase III (EcTOP3) is much more efficient than EcTOP1 in DNA catenation/decatenation, while EcTOP1 can remove negative supercoils much more rapidly. ${ }^{15}$ Since $M$. tuberculosis only has one type of IA topoisomerase enzyme, MtTOP1 is likely to be required for the resolution of single-stranded DNA recombination and replication intermediates. The catenation activity of Mycobacterium smegmatis topoisomerase I has been demonstrated previously with M13 single-stranded DNA. ${ }^{16}$ Since MtTOP1-704t was found to have robust DNA cleavage-religation capabilities, the truncated enzyme was assayed for catenation of single-stranded M13mp18 DNA circles to determine if it can catalyze passage of a single-stranded DNA through the break created by enzyme cleavage on another single-strand. The results (Fig. 4) showed that MtTOP1-T704 has comparable catenation activity to that of full length MtTOP1.

\section{Overall structure of MtTOP1-704t}


The crystal structure of MtTOP1-704t was determined with molecular replacement ${ }^{17}$ and alternative cycles of model building and refinement (Table 1). In the asymmetric unit, there is one MtTOP1-704t as shown in Fig. 5 . The $15 \mathrm{~N}$-terminal residues (A2 to $\mathrm{S} 16$ ) are not visible in the electron density maps of the structure. This indicates the flexible nature of the $\mathrm{N}$-terminal leading peptide, and the flexibility is consistent with its glycine-rich (five Gly residues) composition. Interestingly, the first 15 residues (A2 to S16) of the peptide are "extra" compared to EcTOP1, Fig. 6. Additionally, the leading peptide is positively charged (including one Arg and two Lys residues, Supplementary Fig. 1). The functionality of this leading peptide is currently unknown.

The model of the crystal structure of MtTOP1-704t starts from G17, which corresponds to the starting residue G2 of the EcTOP1 structure (Fig. 6). There is no indication for a stable quaternary assembly based on a PISA (Protein, Interface, Structures, and Assemblies) analysis, ${ }^{18}$ which is consistent with the observation that MtTOP1 is a monomer in solution. The four N-terminal domains (D1-D4, G17-A638) have a similar toroid assembly as EcTOP1 (G2-N590) while the first C-terminal domain D5 shows sequential and structural novelties among known protein folds as discussed later.

To evaluate the similarity of the N-terminal assembly of MtTOP1 to that of EcTOP1, the N-terminal domains of these two molecules were aligned, ${ }^{19}$ resulting in a root mean square deviation (rmsd) of $1.92 \AA$ and sequence identity of $44.9 \%$ (Fig.7A). Pair-wise alignments of individual domains resulted in rmsd values of $1.01 \AA, 1.46 \AA$, $1.35 \AA$, and $1.39 \AA$ for D1, D2, D3, and D4, respectively. Their sequence identities are 
$56.0 \%, 26.9 \%, 49.6 \%$, and $49.2 \%$, respectively. Apparently, D1 is the most conserved while D2 is the least conserved between MtTOP1 and EcTOP1.

Similarly, when the N-terminal region of MtTOP1 is superimposed on EcTOP3

(Fig. 7B), the calculated rmsd value was $3.66 \AA$ with a sequence identity of $21.5 \%$.

Relative domain-domain movement of these enzymes in their crystals could impact the superposition involving multiple domains. Pair-wise individual domain alignments of MtTOP1 and EcTOP3 yielded rmsd values of $1.76 \AA$, $1.93 \AA$, $2.11 \AA$, and $1.85 \AA$ for D1, D2, D3 and D4, respectively. Their sequence identities are $28.3 \%, 19.5 \%, 31.3 \%$, and $25.2 \%$, respectively. From these comparisons it is clear that MtTOP1 is closer to EcTOP1 than EcTOP3. However, MtTOP1 has some local structural features similar to EcTOP3, to be elaborated on later. There is no metal binding observed at the active site of the Apo form of MtTOP1.

\section{Unique features of individual N-terminal domains of MtTOP1}

The D1 or so called TOPRIM (TOpoisomerase-PRIMse) domain is the most conserved domain in the $\mathrm{N}$-terminal region among these enzymes based on the alignments above. In MtTOP1 it includes residues G17 to D157 except the region from R50 to S86, which sticks out from D1 toward D4 (colored grey in Fig. 5). In the EcTOP1 structure, a similar protruding region is defined as a part of D4 because of its close association with the main body of D4 and its partial role in forming a ssDNA-binding groove on D4. ${ }^{20,21}$ For comparison, we define this region in MtTOP1 as a part of D4 as well, although it is described as a part of D1 in the EcTOP3 structure. ${ }^{22}$ In MtTOP1, this protruding region has no sequence homology to the corresponding region in EcTOP1 
and is 15 amino acids shorter (Figs. 5 and 6). Though this protruding region from R50 to $\mathrm{S} 86$ does not have a prominent $\alpha$-helix seen in EcTOP1 (Fig. 7A), it is well structured, comprising five $\beta$ turns and a short $\beta$ hairpin. Interestingly, in EcTOP3, this portion is also a tight loop, similarly consisting of multiple $\beta$ turns $^{22}$ (Fig. 7B). The significance of the structural variation of the protrusion remains unknown.

The hinge domain D2 comprises two sequentially separated regions: F213 to R302 ( $\beta 1-\beta 2-\alpha 1-\beta 3)$ and D434 to R516 ( $\beta 4-\beta 5-\alpha 2-\beta 6)$ (Figs. 5 and 6). Though the sequence identities of the domain between MtTOP1 and EcTOP1 or EcTOP3 are as low as $26.9 \%$ and $19.5 \%$, respectively, the hinge domain of MtTOP1 essentially has a similar structure to that of the two E.coli topoisomerases except for two rather prominent insertions in MtTOP1. The first insertion (T252 to I270, colored magenta in Figs. 5A and 7) creates a large loop between $\beta 2$ and $\alpha 1$ on the right side behind the toroid hole. The magenta loop is rich in charged residues and seemingly creates a positively charged edge on the top of the toroid hole (Fig. 5). A smaller insertion (loop214-255) was found in EcTOP3, which forms a helical motif instead (Fig 7B). ${ }^{22}$ The second insertion (G480 to A485, colored red in Figs. $5 \mathrm{~A}$ and 7 ) is part of a long loop between $\alpha 2$ and $\beta 6$ in the bottom front of the toroid hole. This loop is negatively charged. The two charged loops (colored in magenta and red) positioned on the two sides of the toroid hole of MtTOP1 might play an important coordinating role in the proposed route of the passing strand during catalysis of DNA relaxation by attracting or repelling DNA. The magenta colored insertion T252 to I270 is unique to MtTOP1 and EcTOP3 and may play roles that differentiate MtTOP1 and EcTOP3 from EcTOP1. 
D3 is an all $\alpha$-helical domain (P303 to A433), including the catalytic residue Y342 on a loop between the $\alpha 2$ and $\alpha 3$ helices. The catalytic site of the Apo form of MtTOP1 centered at Y342 shows no significant conformational variation compared to the catalytic sites of the Apo forms of either EcTOP $1^{23}$ or EcTOP3, ${ }^{22}$ indicating the same catalytic mechanism for these type IA topoisomerases. The catalytic site also includes the DxDREG TOPRIM sequence motif from D1, which is quite conserved (Fig. 6). The only difference is the variation in residue $x$, which is Gly112 in MtTOP1, a Leu in EcTOP1, and a Pro in EcTOP3. Additionally, between the a3 and a4 helices of D3, there is a long loop of 35 residues, including the sequence motif Q386EAHEAIRP that is highly conserved in type IA topoisomerases, with the invariant histidine proposed to play a role in catalysis ${ }^{24}$. In all of the EcTOP1 structures determined to date, a part of the loop upstream of the conserved sequence is partially disordered. In MtTOP1, in contrast, this loop is well structured without any break and seemingly correlates to the formation of the $\alpha 4$ helix (highlighted in blue, Fig. 5), which degenerates to a $\beta$-turn in EcTOP1 (Fig. 7A).

In MtTOP1, a feature of D4 may be related to one of its physiological functions; the last helix in D4 (a8) has an insertion of 14 residues (G610 to G623, colored purple in Figs. 5 and 7 ) in the middle of the helix, which is not found in EcTOP1 and EcTOP3. The purple insertion is right below the decatenation loop of EcTOP3, ${ }^{22}$ which is also from D4 (Fig. 7B). The actual functionality of this unusual insertion in the a8 helix needs to be explored. The $a 8$ helix is then followed by a linker to the $\mathrm{C}$-terminal domains. The linker is very different in sequence and structure among MtTOP1, EcTOP1, and EcTOP3, indicating the connection between the $\mathrm{N}$ - and $\mathrm{C}$-terminal domains varies 
significantly among these topoisomerases. However, the DNA-binding groove is mostly conserved (Fig. 5). Two negatively charged sulfate groups likely from the crystallization buffer were identified inside the positively charged DNA-binding groove (not shown). They may mimic two phosphate groups of DNA in their interactions within the groove.

\section{Novel MtTOP1 C-terminal domain}

D5 is the first C-terminal domain, from R638 to P705. This small domain of 67 residues is composed of a four-stranded antiparallel $\beta$-sheet with a C-terminal helix packed against the $\beta$-sheet on one side (Fig. 8). Hydrophobic interaction dominates the interaction between the $\beta$-sheet and the helix. An additional salt bridge (R668-E699) and hydrogen bonds are also observed. The helix apparently plays an important role in stabilizing the $\beta$-sheet and the small domain. The other side of the $\beta$-sheet is open and hosts several residues that may be involved in DNA binding. The conserved tyrosine Y665, the third residue of the $\beta 3$-strand, could be a key residue in the interaction of D5 with DNA by forming m-stacking(s) with the base(s) of DNA (Fig. 5). The other residues Y656 and R658 on the $\beta 2$-strand, R681 on the $\beta 4$-strand and K661 on the sharp $\beta 2-\beta 3$ turn (Fig. 5) can potentially form $\pi$-stacking, $\pi-$ cation, or other forms of interaction with DNA.

A Dali structural homology search ${ }^{25}$ of MtTOP1 results in no significant hits ( $Z$ score $\leq 4.3$, rmsd $\geq 2.6$, and sequence identity $\leq 14 \%$ ) even though a protein fold containing a helix packed against one face of an anti-parallel $\beta$-sheet is common. This indicates the structural novelty of the MtTOP1 C-terminal domain. 


\section{Classification of topoisomerase C-terminal domains.}

From Fig. 1, it is clear that the four MtTOP1 C-terminal domains exemplified by D5 represent one subgroup (Topo_C_Rpt) of bacterial topoisomerase C-terminal repeats. In this subgroup a $\mathrm{C}$-terminal domain has a four-stranded antiparallel $\beta$-sheet compounded or stabilized by a crossing-over $\alpha$-helix on one side of the $\beta$-sheet. Besides MtTOP1, Topo_C_Rpt can also be found in other Mycobacterium species as well as related species of Actinobacteria (Supplementary Fig. 1). The second subgroup of the C-terminal domain of topoisomerases, namely Topo_C_ZnRpt, is represented by the 4-Cys zinc ribbon domains of EcTOP1, in which one four-stranded antiparallel $\beta$ sheet is stabilized by a Zn-binding site on the top of each domain ${ }^{9}$ (PDB ID: $\underline{4 R U L}$ ). Variations of these two subgroups or even new subgroups are expected with further structural characterization of topoisomerases. For example, the only C-terminal domain of Thermotoga maritima topoisomerase I can be regarded as a member of Topo_C_ZnRpt but without Zn binding.

\section{Discussion}

Trapping of the bacterial topoisomerase I covalent complex on cleaved chromosomal DNA has been shown to result in rapid bacterial cell death, validating this class of enzyme as a useful target for novel bactericidal antibiotics ${ }^{26,27}$. The structure of MtTOP1-704t reported here provides details of the active site and its vicinity that would be useful for the discovery of poison-type inhibitors for trapping of the topoisomerase covalent intermediate. Topoisomerase poisons are typically interfacial inhibitors 
interacting with both DNA and the topoisomerase. ${ }^{28}$ Interactions between potential poison-type inhibitors and MtTOP1 protein residues near the active site are needed to confer high binding affinity and selectivity. For example, while m-AMSA has been shown to inhibit M. tuberculosis topoisomerase I and growth of M. tuberculosis, ${ }^{29}$ this compound has much greater selectivity for eukaryotic type II topoisomerases. A binding pocket for functional groups in m-AMSA present near the active site of human type II topoisomerases accounts for the specific interactions with m-AMSA and its effectiveness as an anticancer drug. The construct of MtTOP1-704t could be utilized in the future for co-crystallization of an enzyme-DNA-inhibitor ternary complex to further enable the discovery of new TB therapies.

Catalytic inhibitors of MtTOP1 could also be useful for treating TB by inhibiting the essential function of the enzyme. MtTOP1-704t has no detectable relaxation activity. Some or all other C-terminal parts of MtTOP1 including D6, D7, and D8 or even the flexible tail may be required for relaxation of DNA supercoiling. MtTOP1-704t can carry out DNA cleavage-religation and catalyze the catenation of ssDNA with efficiencies comparable to those of full-length MtTOP1. All Mycobacterium species and most members of the Actinobacteria phylum have only one type IA topoisomerase encoded in their genomes. These include the topoisomerase I with a domain organization similar to MtTOP1 found in the pathogens Corynebacterium diphtheria and Actinomyces odontolyticus (Supplementary Fig. 1) that are likely to also be essential for viability. Unique features observed in the N-terminal domains of MtTOP1, such as the charged loops on opposite sides of the toroid hole and the insertion at the position near where the decatenation loop of EcTOP3 can be found, may also have evolved for 
MtTOP1 to carry out all the catalytic functions that may be associated with bacterial type IA topoisomerases. Further studies are required to determine the biochemical and physiological significance of these unique structural features.

The novel structure of the MtTOP1 C-terminal domain D5 reported here provides new insights into the folding of diverse bacterial topoisomerase I proteins, particularly their C-terminal regions. The organization of repeated bacterial topoisomerase I Cterminal domains may have arisen during evolution through gene duplication. Gene segments that encode a specific protein fold that can interact with ssDNA have been combined in the evolutionary process with the segments encoding the $\mathrm{N}$-terminal domains that have the DNA cleavage-religation active site at the entrance of a toroid hole. Different repeats of the Topo_C_Rpt or Topo_C_ZnRpt domains or their likenesses are utilized in each bacterial species for the DNA-protein or protein-protein interactions that are required for the catalytic activity and physiological functions of this ubiquitous enzyme. Interaction between the C-terminal domains of EcTOP1 and RNA polymerase is important for the efficient removal of transcription-driven negative supercoiling during transcription elongation. ${ }^{30}$ The C-terminal domains of MtTOP1 may interact similarly with $M$. tuberculosis RNA polymerase for relieving the torsional stress generated during transcription.

The flexibility of the linkers between the individual C-terminal domains may account for the difficulty encountered in the crystallization of full-length bacterial topoisomerase I proteins. This could be addressed by introducing a DNA binding substrate or protein interaction partner to limit the degree of interdomain movement, facilitating the crystallization of full length proteins. Successful determination of full- 
length Mycobacterium topoisomerase I crystal structures will provide additional new insights into the mechanism and function of this essential enzyme.

\section{Materials and Methods}

\section{Cloning, expression, and purification}

A clone for expression of MtTOP1-704t was constructed using the Gibson Assembly Master Mix from New England BioLabs, placing the coding sequence of residues 2-704 of MtTOP1 under the control of the T7 promoter in pET-His 6 -Mocr TEVLIC cloning vector (2O-T) that was a gift from Scott Gradia (Addgene plasmid \# 29710). The MtTOP1-704t protein was expressed in the E. coli T7 Express Crystal strain (New England BioLabs). Cells were grown in Luria Bertani broth at $37^{\circ} \mathrm{C}$ to the exponential phase $\left(\mathrm{OD}_{600}=0.4\right)$ and left at room temperature for $20 \mathrm{~min}$. Recombinant protein expression was induced by adding $1 \mathrm{mM}$ IPTG. After overnight induction at $22^{\circ} \mathrm{C}$, the cells were collected and subjected to freeze-thaw lysis in buffer $(20 \mathrm{mM}$ sodium phosphate $\mathrm{pH} 7.4,0.5 \mathrm{M} \mathrm{NaCl}, 20 \mathrm{mM}$ imidazole, $1 \mathrm{mg} / \mathrm{ml}$ lysozyme). The recombinant protein in the soluble lysate was allowed to bind to Ni Sepharose 6 Fast Flow (GE Healthcare) and packed into a column. After extensive washing with buffer (20 mM sodium phosphate $\mathrm{pH} 7.4,0.5 \mathrm{M} \mathrm{NaCl}, 20 \mathrm{mM}$ imidazole), the topoisomerase protein was eluted with buffer (20 mM sodium phosphate, $\mathrm{pH} 7.4,0.5 \mathrm{M} \mathrm{NaCl}, 500 \mathrm{mM}$ imidazole). Following treatment with TEV protease, the protein was passed through the $\mathrm{Ni}$ Sepharose again to remove the $\mathrm{His}_{6}-\mathrm{Mocr}$ tag. Additional purification was achieved with single-stranded DNA cellulose column chromatography (Sigma) using an increasing concentration gradient of $\mathrm{KCl}$ to elute the protein. The final purification step 
utilized an S300 size exclusion chromatography column as described previously. ${ }^{31}$ The purified protein was concentrated to about $85 \mathrm{mg} / \mathrm{ml}$ for crystallization.

\section{Crystallization}

The screening for crystallization conditions was set up with a Mosquito nanoliter liquid handler (TTP LabTech) using the sitting drop vapor diffusion technique in 96-well CrystalQuick plates (Greiner). For each condition, $0.2 \mu \mathrm{l}$ of protein and $0.2 \mu \mathrm{l}$ of crystallization formulation were mixed, and the mixture was equilibrated against $140 \mu \mathrm{l}$ of the crystallization solution in each reservoir well. The crystallization screens used were MCSG-1-4 at $16^{\circ} \mathrm{C}$. Diffraction quality crystals appeared under the following condition: 0.2 M Ammonium Sulfate, 0.1 M Tris: $\mathrm{HCl} \mathrm{pH} \mathrm{8.5,} \mathrm{and} \mathrm{25 \%} \mathrm{(w/v)} \mathrm{PEG3350.}$ Prior to data collection, the crystals were treated with cryoprotectant $(20 \%$ glycerol in crystallization buffer) and cryocooled directly in liquid nitrogen.

\section{X-ray diffraction and structure determination}

Sets of single-wavelength diffraction data were collected at $12.66 \mathrm{keV}$ at $100 \mathrm{~K}$ from cryocooled MtTOP1-704t crystals. All data were obtained at the 19-ID beamline of the Structural Biology Center at the Advanced Photon Source at Argonne National Laboratory using the program SBCcollect. ${ }^{32}$ The intensities of each data set were integrated, scaled, and merged with the HKL3000 program suite ${ }^{33}$ (Table 1). The best data set measured has a resolution limit of $2.52 \AA$. The structures of the MtTOP1-704t crystal were determined using the molecular replacement method. ${ }^{17}$ The best search template used was the structures of the N-terminal domains (D1-D4) of full-length 
EcTOP1 (PDB ID: $\underline{4 R U L})$. Subsequent model rebuilding for the $\mathrm{N}$-terminal domains and building the first C-terminal domain (D5) based on difference maps was performed using the program $\mathrm{COOT}^{34}$. After several alternative cycles of model building and refinement, the final model was refined using the program Phenix.refine ${ }^{35}$ (Table 1). Structural validation was performed using the program MolProbity. ${ }^{36}$

\section{Relaxation activity assay}

The relaxation reaction was carried out in $20 \mu \mathrm{l}$ of $10 \mathrm{mM}$ Tris- $\mathrm{HCl}(\mathrm{pH} 8.0) 50$ $\mathrm{mM} \mathrm{NaCl}, 0.1 \mathrm{mg} / \mathrm{mL}$ gelatin, and $5 \mathrm{mM} \mathrm{MgCl}_{2}$ with $250 \mathrm{ng}$ of supercoiled $\mathrm{pBAD} / \mathrm{Thio}$ plasmid DNA purified by $\mathrm{CsCl}$ gradient centrifugation. Following incubation at $37^{\circ} \mathrm{C}$ for $30 \mathrm{~min}$, the reactions were terminated and analyzed by agarose gel electrophoresis as previously described. ${ }^{37}$

\section{Assay of oligonucleotide DNA cleavage and religation}

The 32 base oligonucleotide substrate 5'-

CAGTGAGCGAGCTTCCGCT $\downarrow$ TGACATCCCAATA-3' with a preferred cleavage site for MtTOP1 indicated by the arrow has been previously described. ${ }^{13}$ It was labeled at the 5'-end with $\mathrm{y}^{-}{ }^{32} \mathrm{P}-\mathrm{ATP}$ and T4 polynucleotide Kinase. Cleavage activity was assayed by incubating the labeled oligonucleotide (0.5 pmole) with MtTOP1 and MtTOP1-704t in 5 $\mu \mathrm{l}$ of $10 \mathrm{mM}$ Tris- $\mathrm{HCl} \mathrm{pH} 8.0$ at $37^{\circ} \mathrm{C}$ for $30 \mathrm{~min}$. Intramolecular religation was initiated by addition of $\mathrm{MgCl}_{2}$ followed by further incubation for $15 \mathrm{~min}$. The reactions were terminated by the addition of an equal volume of sequencing gel loading buffer. The reaction substrate and the 19 base-long cleavage product were separated by 
electrophoresis in a 15\% sequencing gel followed by Phosphor-Imager analysis of the gel.

\section{Catenation activity assay}

Catenation of M13 ssDNA circles was assayed with a protocol similar to previously described conditions ${ }^{16}$. The reaction volume of $20 \mu$ l contained $20 \mathrm{mM}$ Tris$\mathrm{HCl}$ (pH 8.0), $20 \mathrm{mM} \mathrm{KCl}, 2 \mathrm{mM} \mathrm{MgCl}, 0.05 \mathrm{mg} / \mathrm{ml} \mathrm{BSA}, 10 \mathrm{mM}$ spermidine, 30\% glycerol, and $0.5 \mu \mathrm{g}$ of M13 ssDNA (from Bayou Biolabs). After incubation with the indicated amount of topoisomerase protein for $1 \mathrm{~h}$ at $52^{\circ} \mathrm{C}$, the reactions were terminated with the addition of $1 \%$ SDS, $5 \mathrm{mM}$ EDTA, and $12 \mu \mathrm{g}$ of proteinase $\mathrm{K}$, and left at $37^{\circ} \mathrm{C}$ for $1 \mathrm{~h}$ before electrophoresis in $0.8 \%$ agarose gel.

\section{Accession Number}

The atomic coordinates and structure factors of $M$. tuberculosis topoisomerase I have been deposited in the Protein Data Bank with accession code 5D5H.

\section{Acknowledgments}

The authors wish to thank members of the Structural Biology Center at Argonne National Laboratory for their help with data collection at the 19-ID beamline. We are grateful to Ms. Gekleng Chhor for her proofreading. This work was supported by the National Institute of Health grants GM054226 and AI069313 to YT and GM094585 to AJ. The use of SBC 19-ID was supported by the U. S. Department of Energy, Office of Biological and Environmental Research, under contract DE-AC02-06CH11357. 


\section{References}

1. Vos S. M., Tretter E. M., Schmidt B. H. \& Berger J. M. (2011) All tangled up: how cells direct, manage and exploit topoisomerase function. Nat. Rev. Mol. Cell Biol. 12, 827-841. 10.1038/nrm3228; 10.1038/nrm3228.

2. Chen S. H., Chan N. L. \& Hsieh T. S. (2013) New mechanistic and functional insights into DNA topoisomerases. Annu. Rev. Biochem. 82, 139-170. 10.1146/annurevbiochem-061809-100002; 10.1146/annurev-biochem-061809-100002.

3. Forterre P., Gadelle D. (2009) Phylogenomics of DNA topoisomerases: their origin and putative roles in the emergence of modern organisms. Nucleic Acids Res. 37, 679692. $10.1093 /$ nar/gkp032.

4. Dawson R., Diacon A. H., Everitt D., van Niekerk C., Donald P. R., Burger D. A., Schall R., Spigelman M., Conradie A., Eisenach K., Venter A., Ive P., Page-Shipp L., Variava E., Reither K., Ntinginya N. E., Pym A., von Groote-Bidlingmaier F. \& Mendel C. M. (2015) Efficiency and safety of the combination of moxifloxacin, pretomanid (PA824), and pyrazinamide during the first 8 weeks of antituberculosis treatment: a phase 2b, open-label, partly randomised trial in patients with drug-susceptible or drug-resistant pulmonary tuberculosis. Lancet 385, 1738-1747. S0140-6736(14)62002-X [pii].

5. Ahmed W., Menon S., Godbole A. A., Karthik P. V. \& Nagaraja V. (2014) Conditional silencing of topoisomerase I gene of Mycobacterium tuberculosis validates its essentiality for cell survival. FEMS Microbiol. Lett. 353, 116-123. 10.1111/15746968.12412 [doi].

6. Sridevi J. P., Suryadevara P., Janupally R., Sridhar J., Soni V., Anantaraju H. S., Yogeeswari P. \& Sriram D. (2015) Identification of potential Mycobacterium tuberculosis 
topoisomerase I inhibitors: a study against active, dormant and resistant tuberculosis.

Eur. J. Pharm. Sci. 72, 81-92. 10.1016/j.ejps.2015.02.017 [doi].

7. Godbole A. A., Ahmed W., Bhat R. S., Bradley E. K., Ekins S. \& Nagaraja V. (2015) Targeting Mycobacterium tuberculosis topoisomerase I by small-molecule inhibitors. Antimicrob. Agents Chemother. 59, 1549-1557. 10.1128/AAC.04516-14 [doi]. 8. Ravishankar S., Ambady A., Awasthy D., Mudugal N. V., Menasinakai S., Jatheendranath S., Guptha S., Sharma S., Balakrishnan G., Nandishaiah R., Ramachandran V., Eyermann C. J., Reck F., Rudrapatna S., Sambandamurthy V. K. \& Sharma U. K. (2015) Genetic and chemical validation identifies Mycobacterium tuberculosis topoisomerase I as an attractive anti-tubercular target. Tuberculosis (Edinb) S1472-9792(15)20742-1 [pii].

9. Tan K., Zhou Q, Cheng B., Zhang Z., A, Joachimiak A. \& tse-Dinh, Y.C.(2015) Structural basis for suppression of hypernegative DNA supercoiling by E. coli topoisomerase I. Nucleic Acids Res., Oct 20. pii: gkv1073. [Epub ahead of print] 10. Schuster-Bockler B., Schultz J. \& Rahmann S. (2004) HMM Logos for visualization of protein families. BMC Bioinformatics 5, 7. 10.1186/1471-2105-5-7 [doi]. 11. Drozdetskiy A., Cole C., Procter J. \& Barton G. J. (2015) JPred4: a protein secondary structure prediction server. Nucleic Acids Res. 43, W389-94. 10.1093/nar/gkv332 [doi].

12. Hansen G., Harrenga A., Wieland B., Schomburg D. \& Reinemer P. (2006) Crystal structure of full length topoisomerase I from Thermotoga maritima. J. Mol. Biol. 358, 1328-1340. S0022-2836(06)00332-9 [pii]. 
13. Ahmed W., Bhat A. G., Leelaram M. N., Menon S. \& Nagaraja V. (2013) Carboxyl terminal domain basic amino acids of mycobacterial topoisomerase I bind DNA to promote strand passage. Nucleic Acids Res. 41, 7462-7471. 10.1093/nar/gkt506 [doi].

14. Annamalai T., Dani N., Cheng B. \& Tse-Dinh Y. C. (2009) Analysis of DNA relaxation and cleavage activities of recombinant Mycobacterium tuberculosis DNA topoisomerase I from a new expression and purification protocol. BMC Biochem. 10, 18. 10.1186/1471-2091-10-18.

15. Terekhova K., Marko J. F. \& Mondragon A. (2013) Studies of bacterial topoisomerases I and III at the single-molecule level. Biochem. Soc. Trans. 41, 571575. 10.1042/BST20120297 [doi].

16. Bhaduri T., Bagui T. K., Sikder D. \& Nagaraja V. (1998) DNA topoisomerase I from Mycobacterium smegmatis. An enzyme with distinct features. J. Biol. Chem. 273, 13925-13932. .

17. Vagin A., Teplyakov A. (2010) Molecular replacement with MOLREP. Acta Crystallogr. D Biol. Crystallogr. 66, 22-25. 10.1107/S0907444909042589 [doi]. 18. Krissinel E., Henrick K. (2007) Inference of macromolecular assemblies from crystalline state. J. Mol. Biol. 372, 774-797. S0022-2836(07)00642-0 [pii].

19. Krissinel E., Henrick,K. (2004) Secondary-structure matching (SSM), a new tool for fast protein structure alignment in three dimensions. Acta Crystallogr. D Biol. Crystallogr., 60, 2256-2268.

20. Perry K., Mondragon A. (2003) Structure of a complex between E. coli DNA topoisomerase I and single-stranded DNA. Structure 11, 1349-1358. . 
21. Cheng B., Feng J., Mulay V., Gadgil S. \& Tse-Dinh Y. C. (2004) Site-directed mutagenesis of residues involved in G Strand DNA binding by Escherichia coli DNA topoisomerase I. J. Biol. Chem. 279, 39207-39213. 10.1074/jbc.M405891200.

22. Mondragon A., DiGate R. (1999) The structure of Escherichia coli DNA topoisomerase III. Structure 7, 1373-1383. st7b02 [pii].

23. Lima C.D., Wang J.C. \& MondragonA. (1994) Three-dimensional structure of the $67 \mathrm{~K} \mathrm{~N}$-terminal fragment of E. coli DNA topoisomerase I. Nature, 367, 138-146.

24. Perry K., Mondragon A. (2002) Biochemical characterization of an invariant histidine involved in Escherichia coli DNA topoisomerase I catalysis. J. Biol. Chem. 277, 13237-13245. 10.1074/jbc.M112019200.

25. Holm L., Rosenstrom P. (2010) Dali server: conservation mapping in 3D. Nucleic Acids Res. 38, W545-9. 10.1093/nar/gkq366 [doi].

26. Tse-Dinh Y. C. (2015) Targeting bacterial topoisomerase I to meet the challenge of finding new antibiotics. Future Med. Chem. 7, 459-471. 10.4155/fmc.14.157 [doi]. 27. Tse-Dinh Y. C. (2009) Bacterial topoisomerase I as a target for discovery of antibacterial compounds. Nucleic Acids Res. 37, 731-737. 10.1093/nar/gkn936. 28. Pommier Y. (2013) Drugging topoisomerases: lessons and challenges. ACS Chem. Biol. 8, 82-95. 10.1021/cb300648v; 10.1021/cb300648v.

29. Godbole A. A., Ahmed W., Bhat R. S., Bradley E. K., Ekins S. \& Nagaraja V. (2014) Inhibition of Mycobacterium tuberculosis topoisomerase I by m-AMSA, a eukaryotic type II topoisomerase poison. Biochem. Biophys. Res. Commun. 446, 916920. 10.1016/j.bbrc.2014.03.029 [doi]. 
30. Cheng B., Zhu C. X., Ji C., Ahumada A. \& Tse-Dinh Y. C. (2003) Direct interaction between Escherichia coli RNA polymerase and the zinc ribbon domains of DNA topoisomerase I. J. Biol. Chem. 278, 30705-30710. 10.1074/jbc.M303403200 [doi]; M303403200 [pii].

31. Zhang Z., Cheng B. \& Tse-Dinh Y. C. (2011) Crystal structure of a covalent intermediate in DNA cleavage and rejoining by Escherichia coli DNA topoisomerase I. Proc. Natl. Acad. Sci. U. S. A. 108, 6939-6944. 10.1073/pnas.1100300108.

32. Rosenbaum G., Alkire R. W., Evans G., Rotella F. J., Lazarski K., Zhang R. G., Ginell S. L., Duke N., Naday I., Lazarz J., Molitsky M. J., Keefe L., Gonczy J., Rock L., Sanishvili R., Walsh M. A., Westbrook E. \& Joachimiak A. (2006) The Structural Biology Center 19ID undulator beamline: facility specifications and protein crystallographic results. J. Synchrotron Radiat. 13, 30-45. S0909049505036721 [pii].

33. Minor W., Cymborowski M., Otwinowski Z. \& Chruszcz M. (2006) HKL-3000: the integration of data reduction and structure solution--from diffraction images to an initial model in minutes. Acta Crystallogr. D Biol. Crystallogr. 62, 859-866.

10.1107/S0907444906019949.

34. Emsley P., Lohkamp B., Scott W. G. \& Cowtan K. (2010) Features and development of Coot. Acta Crystallogr. D Biol. Crystallogr. 66, 486-501. 10.1107/S0907444910007493.

35. Afonine P. V., Grosse-Kunstleve R. W., Echols N., Headd J. J., Moriarty N. W., Mustyakimov M., Terwilliger T. C., Urzhumtsev A., Zwart P. H. \& Adams P. D. (2012) Towards automated crystallographic structure refinement with phenix.refine. Acta Crystallogr. D Biol. Crystallogr. 68, 352-367. 10.1107/S0907444912001308 [doi]. 
36. Davis I. W., Leaver-Fay A., Chen V. B., Block J. N., Kapral G. J., Wang X., Murray L. W., Arendall W. B.,3rd, Snoeyink J., Richardson J. S. \& Richardson D. C. (2007) MolProbity: all-atom contacts and structure validation for proteins and nucleic acids. Nucleic Acids Res. 35, W375-83. gkm216 [pii].

37. Zhu C. X., Tse-Dinh Y. C. (2000) The acidic triad conserved in type IA DNA topoisomerases is required for binding of $\mathrm{Mg}(\mathrm{II})$ and subsequent conformational change. J. Biol. Chem. 275, 5318-5322. 


\section{Figure Legends}

Fig. 1. Division of the C-terminal region of M. tuberculosis topoisomerase I into four domains and a flexible tail. The conserved residues among the four domains are highlighted in red, including the GxxGPY sequence motif. Besides the conserved tyrosine residues within the GxxGPY motifs, other possible DNA binding residues are highlighted in magenta (for possible $\pi$ stacking to the bases of nucleotides) or blue (for possible $\pi$-cation or salt bridge or hydrogen bond to DNA). The secondary structures of D5 are indicated above the appropriate sequence of D5. The highly positively charged tail after D8 is listed below the alignment, and it is presumably structurally flexible.

Fig. 2. Assay of relaxation activity of full length MtTOP1 and MtTOP1-704t. Supercoiled pBAD/Thio plasmid DNA was incubated with the indicated amount of MtTOP1 or MtTOP1-704t at $37^{\circ} \mathrm{C}$ for 30 min before separation of the supercoiled DNA from relaxed topoisomers by agarose gel electrophoresis.

Fig. 3. DNA cleavage and religation by MtTOP1-704t (A) Comparison of the formation of DNA cleavage product by MtTOP1 and MtTOP1-704t. O: Oligonucleotide substrate; P: Major cleavage product. (B) Intramolecular DNA religation by MtTOP1 and MtTOP1-704t. Lane 1: Oligonucleotide substrate with no enzyme; Lanes 2 and 5: DNA cleavage reaction. (C) DNA religation $(\mathrm{R})$ with no $\mathrm{Mg}^{2+}$ present; Lanes 3 and 6: 
DNA religation (R) with $5 \mathrm{mM} \mathrm{MgCl}_{2}$ and $1 \mathrm{M} \mathrm{NaCl}$ added; Lanes 4 and 7: DNA religation (R) with $10 \mathrm{mM} \mathrm{MgCl}_{2}$ and $1 \mathrm{M} \mathrm{NaCl}$ added.

Fig. 4. Catenation of single-stranded M13mp18 DNA by MtTOP1 and MtTOP1-704t. Single-stranded M13mp18 DNA was incubated with the indicated amount of MtTOP1 or MtTOP1-704t before proteinase $\mathrm{K}$ treatment. C: circular ssDNA; L: linear ssDNA; CC: catenated circular ssDNA.

Fig. 5. Structure of MtTOP1-704t. (A) A ribbon diagram of the structure of MtTOP1704t. The main bodies of the N-terminal D1, D2, D3, and D4 domains and the Cterminal D5 domain are colored in salmon, yellow, cyan, green, and pink, respectively. Numbering of the secondary structures are based on each individual domain. The protrusion from D1 that is regarded as a part of D4, as described in the EcTOP1 structure, is colored in grey. In D2, the two loops that include unique insertions are colored in magenta (the loop between $\beta 2$ and $\alpha 1$ ) and red (the loop between $\alpha 2$ and $\beta 3)$. In D3, the $\alpha 4$ helix that degenerated to a $\beta$-turn in the EcTOP1 structures is highlighted in blue. The catalytic residue Y342 is drawn in stick format. In D4, the unusual insertion in the middle of the $a 8$ helix is highlighted in purple. (B) and (C) The molecular surface representations of MtTOP1-704t. The orientation of the molecule in $(B)$ is the same as that in $(A)$. The orientation of the molecule in $(C)$ has a $180^{\circ}$ rotation around the vertical axis with respect to the view in (B). (D) and (E) The electrostatic potential surface representations of MtTOP1-704t. The surface of MtTOP1-704t is colored according to amino acid residue charge, ranging from blue (positive) to red 
(negative). The orientation of the molecule in (D) is similar to that in $(B)$, but with a $-15^{\circ}$ rotation around the horizontal axis with respect to the view in $(B)$ for a better view of the DNA binding groove. The orientation of the molecule in $(E)$ is the same as that in $(C)$. Figures 5, 7, and 8 are prepared with the program PyMOL (http://www.PyMOL.org).

Fig. 6. N-terminal domain arrangement of MtTOP1 and multiple sequence

alignment. (A) The N-terminal domain arrangement of MtTOP1. Domains D1, D2, D3, and D4 are colored in salmon, yellow, cyan, and green, respectively. The protruded region from D1 in MtTOP1 and EcTOP1, is regarded as a part of D4. In EcTOP3, it is described as a part of $\mathrm{D} 1 .^{22}$ The asterisk indicates the location of the catalytic tyrosine residue. (B) Structure-based Multiple Sequence Alignment with EcTOP1 and EcTOP3. The residues that are identical between MtTOP1 and EcTOP1 are colored in red. The residues of EcTOP3 that are conserved in both MtTOP1 and EcTOP1 are also colored in red. The residues in regions of similar secondary structures but different conformations between MtTOP1 and EcTOP1 are colored in green. The residues in regions with neither sequential nor structural alignments are colored in blue. The disordered residues in the structures of EcTOP1 are shown in lower case. The secondary structures of MtTOP1, $\alpha$-helices as coils and $\beta$-strands as arrows, are drawn above the appropriate sequence of MtTOP1. The numbering of these secondary structures is based on each domain for descriptive convenience. The same color schemes for domains used in $(A)$ are used for highlighting their corresponding sequences of MtTOP1 and EcTOP1 in the structural alignment. 
Fig. 7. N-terminal region structural alignments of MtTOP1 with EcTOP1 and EcTOP3. (A) Ribbon diagrams of the superposition of MtTOP1 with EcTOP1 in a front view and in a side view. The color scheme for the MtTOP1 domains and featured structural parts is the same as in Fig. 5. EcTOP1 is colored in grey. (B) Ribbon diagrams of the superposition of MtTOP1 with EcTOP3 in a front view and in a side view. EcTOP3 is colored in grey, and its decatenation loop is labeled.

Fig. 8. Structure of the first MtTOP1 C-terminal domain (D5). (A) A ribbon diagram of D5. The residues (G660, G663, and Y665) that are part of the highly conserved sequence motif GxxGPY in each MtTOP1 C-terminal domain are highlighted in magenta. Several residues (including Y665) that are potentially involved in DNA binding are drawn in stick format. (B) A stereo view of the $\beta$-sheet of D5 and several exposed residues on the $\beta$-sheet mentioned in (A). The electron density drawn in grey mesh is calculated from a weighted 2Fo-Fc map and contoured at the $1 \sigma$ level. 
Table 1: Data collection and refinement statistics

\begin{tabular}{|c|c|}
\hline Data collection & MtTOP1 \\
\hline Space group & $P 2_{1} 2_{1} 2_{1}$ \\
\hline \multicolumn{2}{|l|}{ Unit Cell dimensions } \\
\hline$a, b, \mathrm{c}(\AA)$ & $64.00,93.15,140.2$ \\
\hline$\alpha, \beta, \gamma\left({ }^{\circ}\right)$ & $90,90,90$ \\
\hline MW Da (residue) & $77,468.3(703)^{1}$ \\
\hline Mol (AU) & 1 \\
\hline Wavelength $(\AA)$ & 0.9792 \\
\hline Resolution $(\AA)$ & $42.0-2.52$ \\
\hline Number of Unique reflections & 28,858 \\
\hline Completeness (\%) & $99.2(97.6)^{2}$ \\
\hline Redundancy & $5.6(5.6)^{2}$ \\
\hline $\mathrm{R}_{\text {merge }}$ & $0.098(0.696)^{2}$ \\
\hline $\mathrm{I} / \sigma(\mathrm{I})$ & $14.0(2.0)^{2}$ \\
\hline Solvent content (\%) & 54.3 \\
\hline \multicolumn{2}{|l|}{ Phasing } \\
\hline Resolution $(\AA ̊)$ & $42.0-3.00$ \\
\hline Correlation coefficient $^{3}$ & 0.416 \\
\hline \multicolumn{2}{|l|}{ Refinement } \\
\hline Resolution & $42.0-2.52$ \\
\hline No. reflections (work/test) & $26,111 / 1,380$ \\
\hline $\mathrm{R}_{\text {work }} / \mathrm{R}_{\text {free }}$ & $0.174 / 0.241$ \\
\hline \multicolumn{2}{|l|}{ No.of atoms } \\
\hline Protein & 5334 \\
\hline Water/Others & $72 / 47$ \\
\hline \multicolumn{2}{|l|}{ B-factors $\left(\AA^{2}\right)$} \\
\hline Protein & 55.0 \\
\hline Water/Others & $40.7 / 77.47$ \\
\hline \multicolumn{2}{|l|}{ R.m.s deviation } \\
\hline Bond length $(\AA)$ & 0.008 \\
\hline Bond angle $\left({ }^{\circ}\right)$ & 1.083 \\
\hline PDB code & $5 \mathrm{D} 5 \mathrm{H}$ \\
\hline
\end{tabular}

${ }^{1}$ Not including three $\mathrm{N}$-terminal vector-derived residues, SNA; ${ }^{2}$ (Last resolution bin, 2.52-2.56 $\AA) ;{ }^{3}$ Molecular replacement 
Figure 1

Click here to download high resolution image

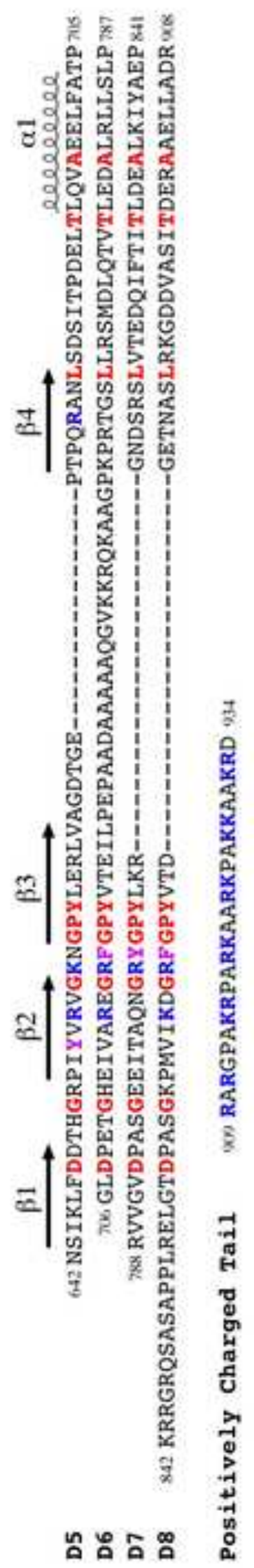




\section{MtTOP1}

No enzyme

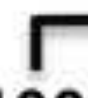

100

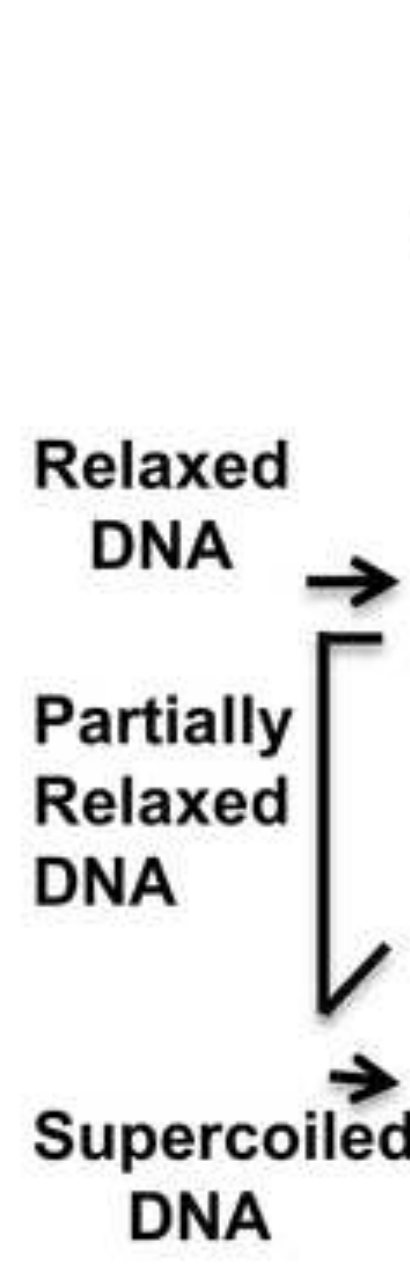

MtTOP1-704t

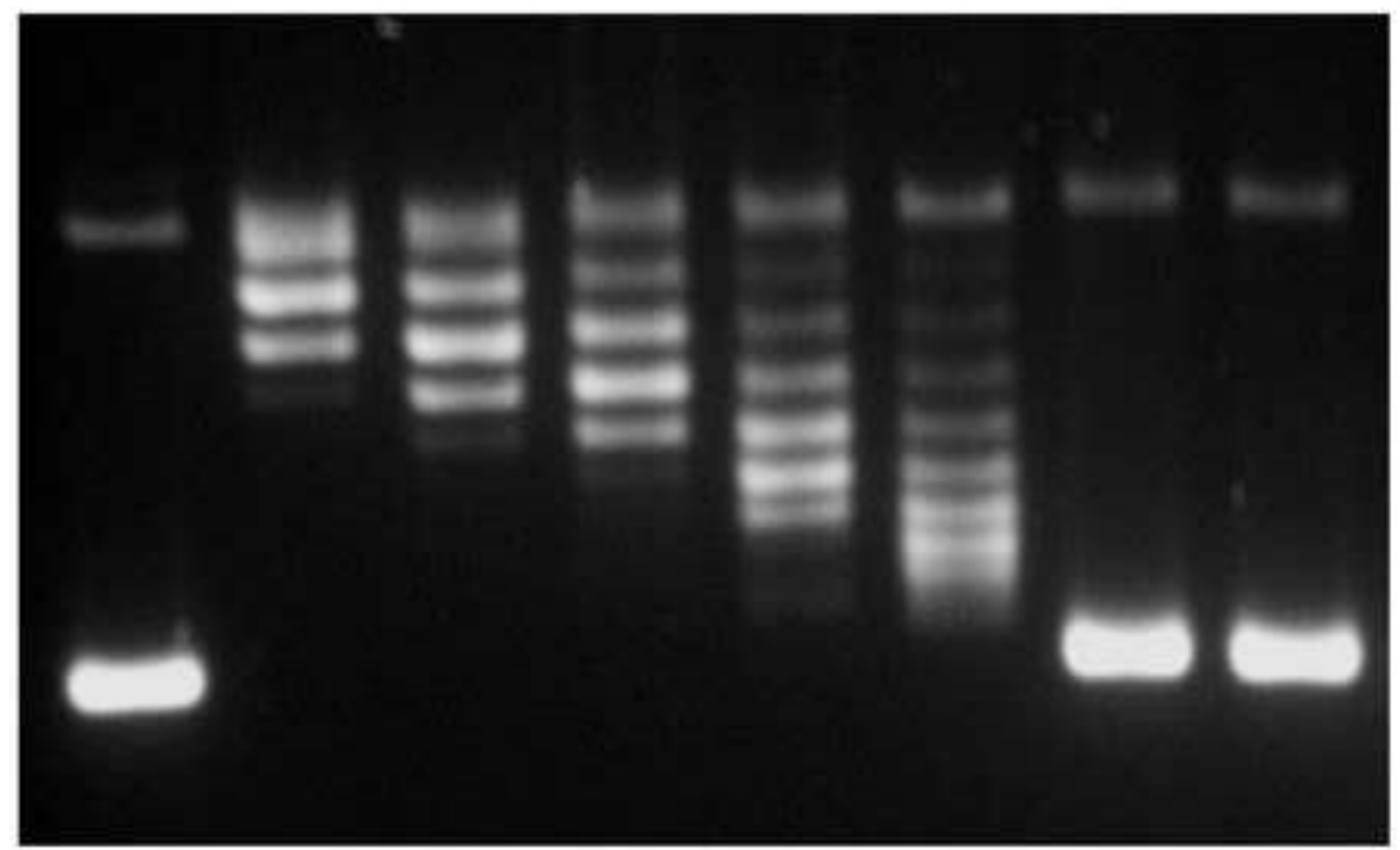


Figure 3

A

\section{DNA Cleavage} MtTOP1

MtTOP1-704t

$\begin{array}{lllllllll}0 & .03 .06 .12 .25 .5 & 1.0 & 0 & .03 .06 .12 .25 .5 & 1 & \text { pmole }\end{array}$

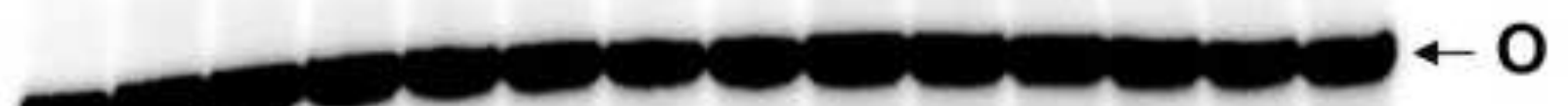

No Enz

\section{MtTOP1 MtTOP1-704t}

$\begin{array}{lllllll}C & R & R & C & R & R\end{array}$

$\begin{array}{llllllll}0 & 5 & 10 & 0 & 5 & 10 & \mathrm{mM} \mathrm{Mg}\end{array}$

$\leftarrow \mathrm{O}$

$\leftarrow \mathbf{P}$

$\begin{array}{lllllll}1 & 2 & 3 & 4 & 5 & 6 & 7\end{array}$ 


\section{MtTOP1}

$\begin{array}{lll}0.5 & 0.25 & 0.125\end{array}$
MtTOP1-704t

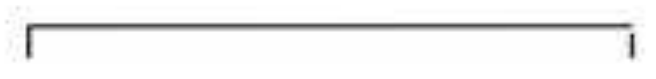

$0.5 \quad 0.25 \quad 0.125$ pmole

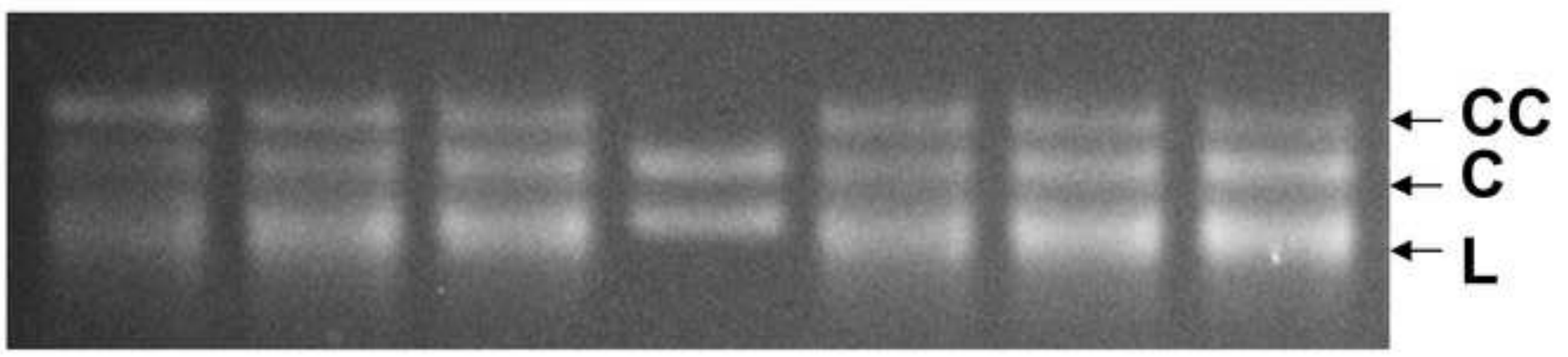


Click here to download high resolution image

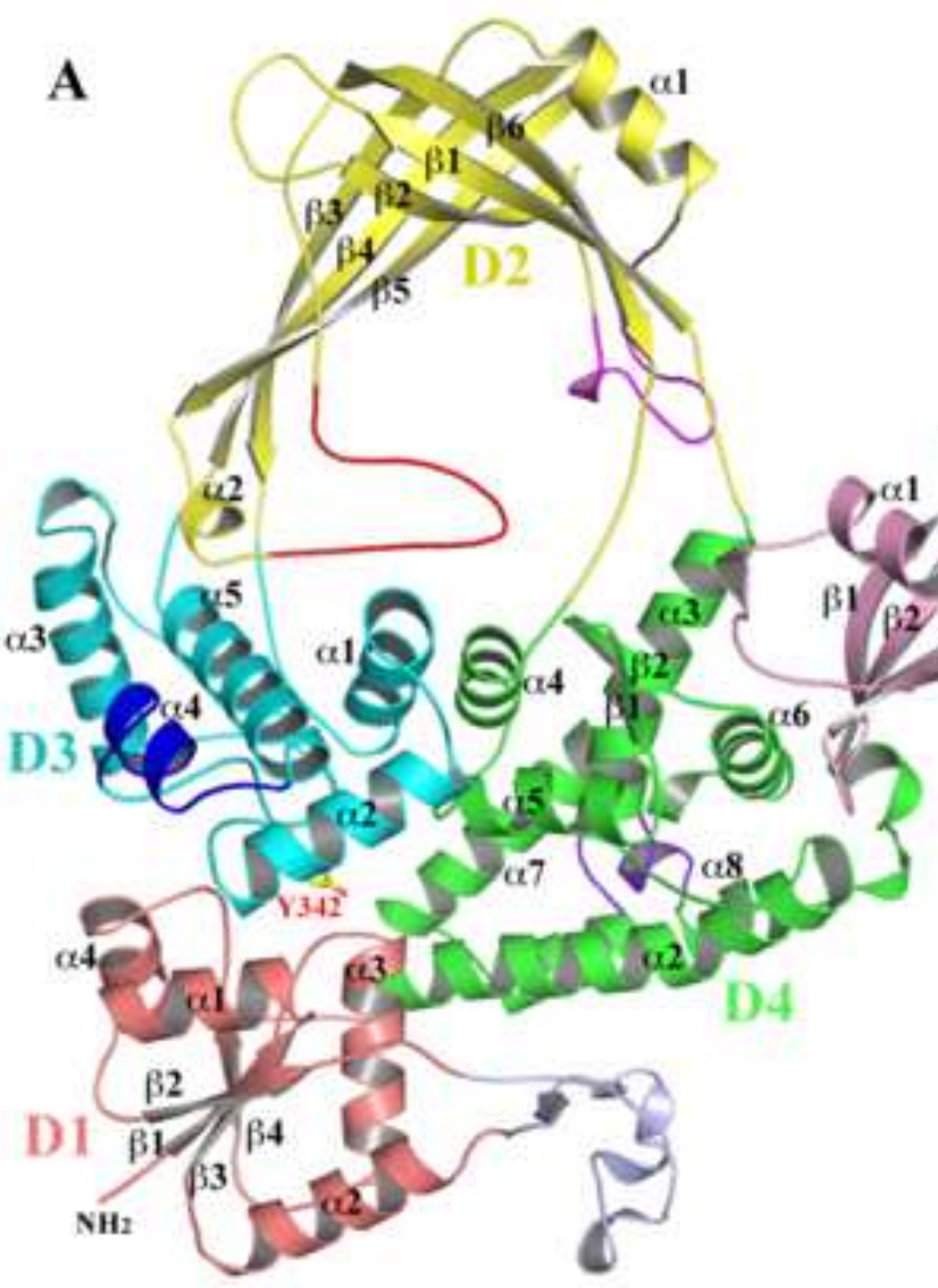

C
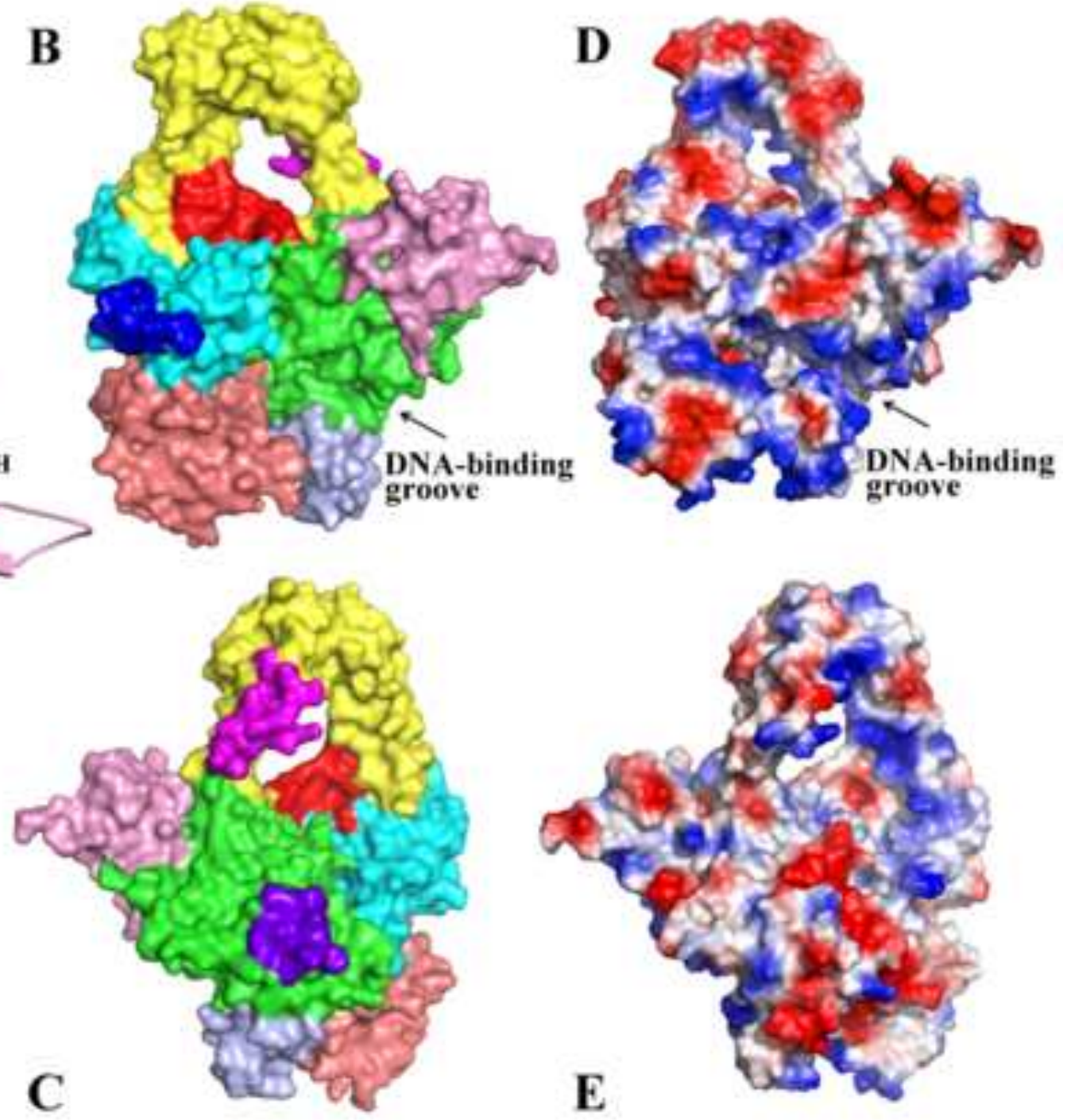

$\mathbf{E}$ 
A N-terminal domain arragement of MtTop1

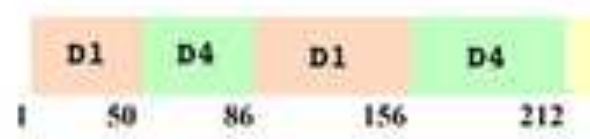

D2

302

\section{$\frac{1342}{25}$}

D3
D2

516
D4
B
17
$\stackrel{11}{\longrightarrow}$ 2ereereere2

$\stackrel{\mathrm{N13}}{\longrightarrow}$
433
516

60

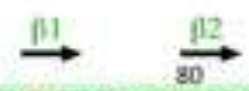

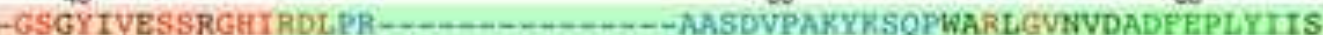

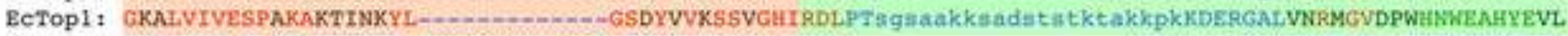
EcTop3: MRLFIAEKPSLARAIADVLPKPHAKGDGEIECGNGQVVTWCIGHLLEQ- -
seeverees
eqeeregereese
$\longrightarrow$
100

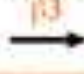
140 eecerever

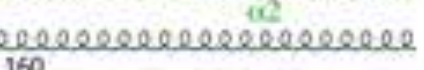

MtTop 1: PEKRSTUSELRGLLKDVDELYLATDGDREGEATAWHLLETLKPR---IPVKRMVFHEITEPAIRAAAEHPRDL-DIDLVDAOETRRILDRLYGYEV ECTOP1: PGKEKVUSELKQLAEKADHIYLATDLDREGEAIAWHLREVIGGD---DARYSRVVENEITKARIRQXFMKPGEL-NIDRVAAOOARRFMDRVVGYMV EcTOp3; PSVTKQLNVIKRFLHEASEIVHAGDPDREGQLLVDEVLDYLQLAPEKROQVQRCLINDLNPQAVERAIDRLRSNSEFVPLCVSALARARADWLYGINM
eeceed
reverequevereveree
$\underset{220}{\stackrel{\rho 1}{\longrightarrow}}$
$\frac{1}{240}$
260

MtTop1: SPVIWKK-_-VAPK-LSAGRVQSVATRIIVARBRDRMARRSAAYWDILAKLDASUSDPDAAPPTPSARLTAVAGRRVATGRDPDSLGTLRKGDEVIV

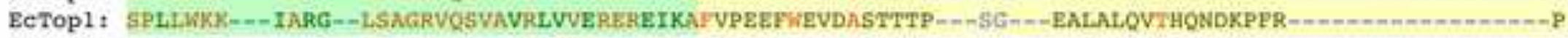
ECTOP3: TRAYTILGRNAGYQGVLSVGRVQTPVLGLVVRRDEEIENEVAKDFPEVKAHIVTP---AD---ERFTAIWQ--------PSEAACEPYQDEEGR---L

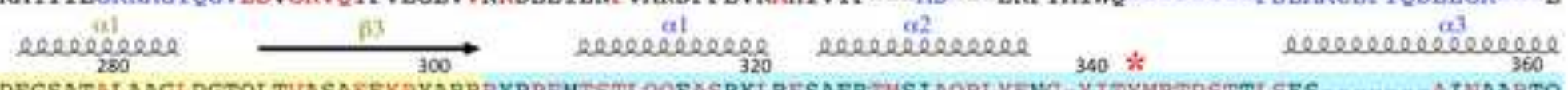

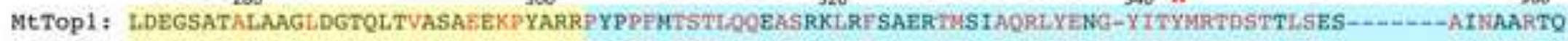

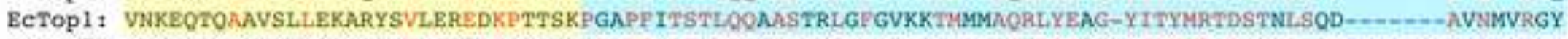
ECTOP 3: LHRPLAEHVVNRISGQPAIVTSYNDKRESESAPLPFSLSALQTEAAKRFGLSAQNVLDICQKLYETHKLTTYPRSDCRYLPEEHFAGRHAVMNAISVH 20200
300
400 erếee

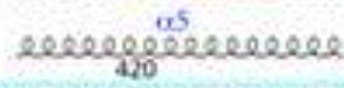 440
MtTOP1: ARQLYCDEYVAPAPROYTRKVKWMOEAHEAIRPAGETFNTPDAVRREIDGPNIODFRLYELTWORTVASQYADARGMTLSLRITGMSGHOEVVPSNTCG

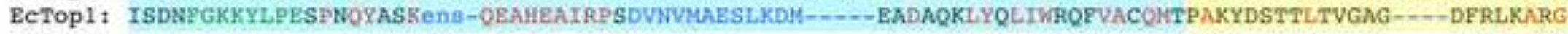
ECTOP3; APDLLPQPVVDPDIRWRCWDDKK-VDAHHAIIPTAFSSAIHL_-.-.--TENEAKVYNLIARQYLMQFCPDAVFRKCVIELDIA----KGKFVAKA
$\longrightarrow 2000$

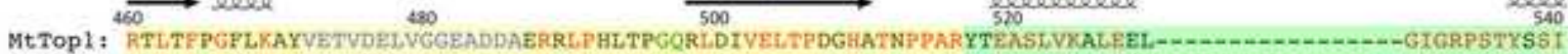

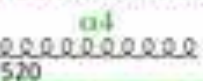
$\frac{2020}{540}$

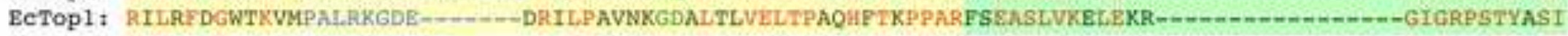

ECTOP3: RFLAEAGWRTLLGSKERDEEND----GTPLPVVAKGDELICEKGEVVERQTQPPRHFTDATLLSAMTGIARFVQDKDLKKILRATDGLGTEATRAGI

MLTop1: IKTIODRCYVHAKGSALVPSWVAFAVTGLLEQHFGRLVDXDTMAMEDELDEIAAGNERRTNWLNWFYFGGDHGVPDSVARSCGLKKLVGTNLEGIDAREV

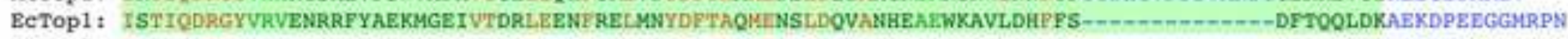
ECTOP 3; IELLFKRGFLTKKGRYIHSTDAGKALFHSL---PEMATRPDMTAHWESVLTQISEKQCRYQDFMQPLVG- 

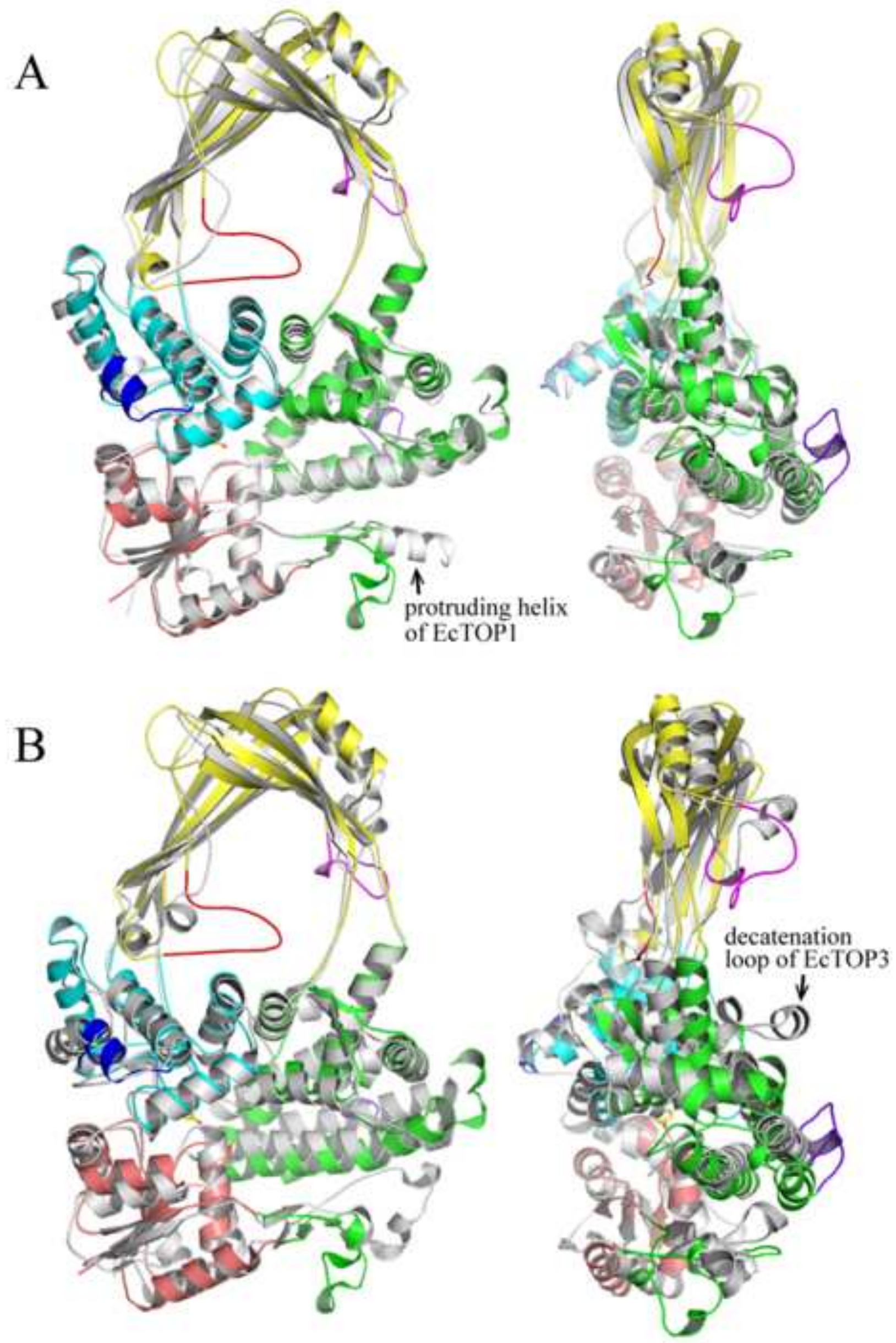

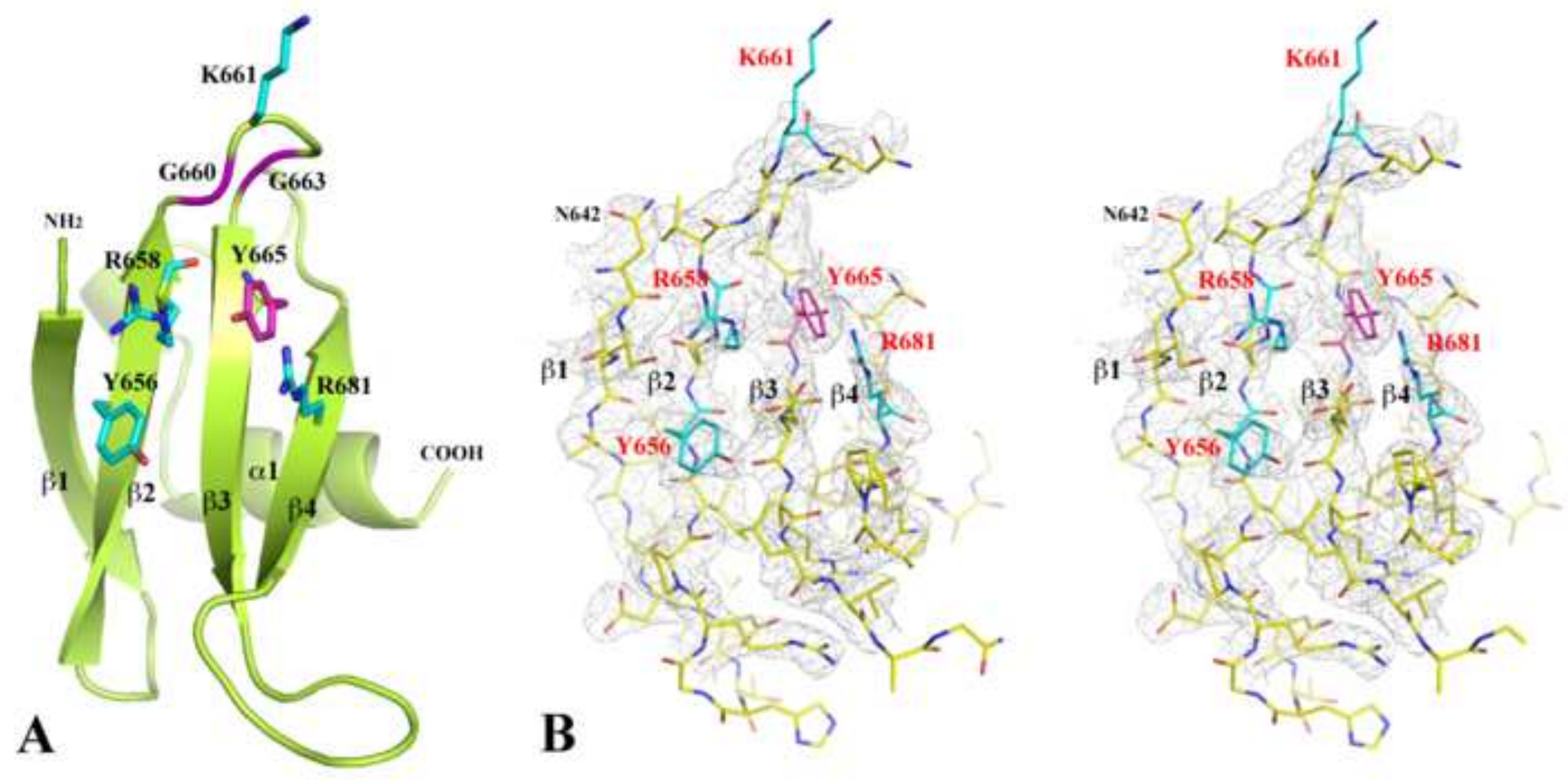


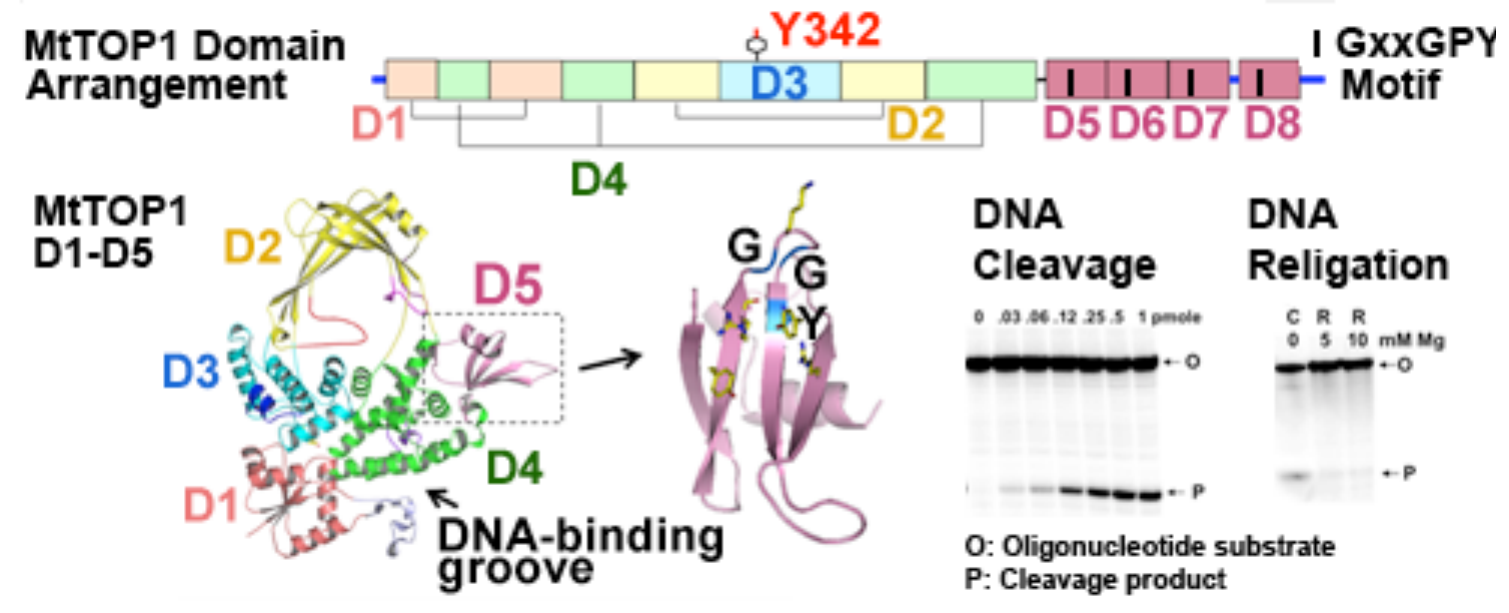

graphical abstract 